\title{
A mathematical optimisation approach for wastewater minimisation in multipurpose batch plants: Multiple contaminants
}

\author{
Thokozani Majozi ${ }^{a}, b, *$, Jacques F. Gouws ${ }^{a}$ \\ a Department of Chemical Engineering, University of Pretoria, Lynnwood Road, Pretoria 0002, South Africa \\ ${ }^{\mathrm{b}}$ Department of Computer Science, University of Pannonia, Egyetem u. 10, Veszprém H-8200, Hungary
}

\section{A R T I C L E I N F O}

\section{Article history:}

Received 31 August 2006

Received in revised form 17 May 2009

Accepted 4 June 2009

\section{Keywords:}

Multiple contaminants

Batch

Optimisation

Wastewater minimisation

\begin{abstract}
A B S T R A C T
This paper presents a methodology for wastewater minimisation in multipurpose batch plants characterised by multiple contaminant streams. Firstly a situation in which central reusable water storage does not exist is considered. In this case, water from one operation is directly reused in another operation as long as the source and sink operations, respectively, end and begin simultaneously. Secondly, the case with central reusable water storage is considered. In this case water from a source operation can temporarily be stored in dedicated storage before use by the sink process. The methodology is based on an existing scheduling framework which then makes it possible to generate the required schedule to realise the absolute minimum wastewater generation for a problem. The methodology involves a two-step solution procedure. In the first step the resulting MINLP problem is linearised and solved to provide a starting point for the exact MINLP problem.
\end{abstract}

(c) 2009 Elsevier Ltd. All rights reserved.

\section{Introduction}

As environmental regulations become ever more stringent and the cost of fresh water increases, the need for production facilities that produce the least amount of effluent water becomes greater. In the past the main focus of research has been on developing methodologies to reduce wastewater in continuous processes. The main driving force behind this is that continuous processes produce much larger volumes of wastewater than batch processes. Moreover, the time implications that have to be addressed due to the discontinuous nature of batch processes are completely overridden in continuous processes.

Wastewater generated from a batch operation is usually characterised by high toxicity levels due to the nature of batch plants, e.g. pharmaceuticals and agrochemicals. However, the volume of water is relatively small. The high toxicity levels make the minimisation of wastewater from batch processes important. Wastewater is generally generated during operations such as liquid-liquid extraction and cleaning of process vessels. As most processes deal with more than one chemical during their operation, wastewater generated from most processes usually have more than one contaminant present. Existence of multiple contaminants instead of just a single contaminant adds further constraints to wastewater minimisation problems.

The inherent discontinuous nature of batch processes complicates the development of a methodology to minimise wastewater, since the methodology not only has to take inlet and outlet concentrations into consideration, but also the times at which water is used during the operation. Many methodologies have been developed to deal with the minimisation of wastewater in continuous processes. The methods proposed can be divided into graphical (Hallale, 2002; Olesen \& Polley, 1997; Wang \& Smith, 1994, 1995a) and mathematical (Alva-Argáez, Kokossis, \& Smith, 1998; Doyle \& Smith, 1997; Jödicke, Fischer, \& Hungerbühler, 2001; Savelski \& Bagajewicz, 2000) methodologies. Graphical methodologies have inherent difficulties when dealing with multiple contaminants, due to the multiple dimensions introduced. On the other hand multiple contaminants can be incorporated easier into mathematical methodologies, since they are fundamentally not limited in dimension.

In the past 10 years, there have been several attempts to adapt wastewater minimisation techniques to batch processes. Similar to their continuous counterparts these attempts can also be categorised into graphical and mathematical techniques. A few contributions in graphical methodologies involve the work of Wang and Smith (1995b), Foo, Manan, and Tan (2005) and Majozi, Brouckaert, and Buckley (2006).

\footnotetext{
* Corresponding author at: Department of Chemical Engineering, University of Pretoria, Lynnwood Road, Pretoria 0002 , South Africa. Tel.: +27 12 420 4130; fax: +27 123625173 .

E-mail addresses: majozi@dcs.vein.hu, thoko.majozi@up.ac.za (T. Majozi).
} 
Wang and Smith (1995b) extended their earlier method for continuous processes (Wang and Smith, 1994) to discontinuous processes. The method involved the sequential targeting of different concentration intervals and the usage of storage tanks to overcome the discontinuity of the processes involved. The method proposed by Wang and Smith (1995b) is, however, restricted to semi-continuous processes since it is inherently assumed that water is flowing throughout an operation. Majozi et al. (2006) derived a method where it is assumed that water is only required and available at the beginning and ending of an operation. Two different targeting methods were proposed by either taking time as the main constraint or concentration as the main constraint. The method derived by Foo et al. (2005) utilised a novel technique called time dependent water cascade analysis, which is based on an earlier method for mass integration in batch processes (Foo, Manan, Yunus, \& Aziz, 2004). Each of the methods described above have the common problem of not being able to effectively deal with multiple contaminant systems as they are derived for single contaminant systems. Furthermore, graphical techniques are all based on the schedule being determined a priori. This means that each time the schedule changes the minimum wastewater target has to be determined from the beginning. Based on practical experience at a pharmaceuticals manufacturing facility, where the schedule changes on a daily basis, this would be highly impractical.

Mathematical based techniques for wastewater minimisation in batch processes have been seen as a means to address the multidimensionality of the problem. The mathematical based methods can be divided into two groups, namely, those based on the schedule being known a priori and those that minimise wastewater generation and determine schedule co-currently. Almató, Sanmartí, Espuña, and Puigjaner (1997) and Kim and Smith (2004) proposed methodologies based on the optimal schedule being known a priori. The method proposed by Almató et al. (1997) utilizes intermediate water storage tanks to overcome the discontinuity of the process. The main aim of the methodology is determine the optimal tank-to-stream assignment. The resulting formulation is a non-linear program, which the authors solved using a heuristic based method. The global optimality of the solution resulting solution is therefore not known. The method proposed by Kim and Smith (2004) addresses multiple contaminant wastewater minimisation and the optimal design of the resulting water network.

As with previous work, Kim and Smith (2004) assume that water is constantly used and consumed during an operation. The proposed method divides the problem into a number of time intervals in which the mass balances of the active units are solved and optimized for water usage. The resulting formulation is a mixed integer non-linear program (MINLP). The authors propose a MILP and LP decomposition strategy to find a starting solution for the MINLP. The global optimality of the resulting solution can, therefore, not be determined. Furthermore, the optimisation of a water reuse network for a fixed schedule is impractical, since the schedule will invariably change at some point during the plants lifetime. One, therefore, has a highly inflexible reuse structure. The methods described above suffer from a common drawback of being based on a fixed plant schedule. True optimality can only be realised if scheduling and wastewater minimisation are optimised simultaneously. Majozi (2005) and Gouws, Majozi, and Gadalla (2008) developed methods that optimize the wastewater target and schedule simultaneously.

The method developed by Majozi (2005) focuses on the single contaminant wastewater minimisation problem. It is based on the scheduling framework developed by Majozi and Zhu (2001) and aims to address the problem where there is a central wastewater storage vessel and where the central storage vessel is not present. On the other hand, the method derived by Gouws et al. (2008) deals with the situation where neither the water flowrate or outlet concentration in any of the operations of interest is fixed. This method uses a two-step solution procedure, which involves the solving of an MILP and then an MINLP. The method is however restricted to single contaminants.

Multiple contaminant wastewater minimisation in batch processes has not been effectively dealt with in previous methodologies. The methods that have dealt with this topic in the past are based on the schedule being known a priori and are more suited to semi-continuous processes. Most methodologies proposed for wastewater minimisation in batch processes deal with the single contaminant scenario, which in essence is not a common occurrence.

The methodology presented in this paper deals with wastewater minimisation in batch processes with multiple contaminants as none of the methodologies developed thus far have dealt with this effectively. The methodology is, in essence, a natural progression of the method developed by Majozi (2005). Two distinct scenarios are dealt with, the first being the scenario where there is no storage available for the wastewater and the second where there is a central storage vessel, with limited capacity, for the wastewater. The solution procedure proposed by Gouws et al. (2008) is adopted to deal with the highly non-linear resulting formulations.

\section{Problem statement}

The problem considered in this paper can be stated as follows.

Given:

(i) the contaminant mass load of each contaminant,

(ii) the necessary cost and stoichiometric data,

(iii) the maximum inlet and outlet concentrations of each contaminant,

(iv) the available units and their capacities,

(v) the time horizon of interest and

(vi) the maximum storage available for water reuse,

determine the production schedule that achieves the minimum amount of wastewater generation through reuse and recycle. Reuse refers to the usage of the outlet water stream from unit $j$ in unit $j$ ' and recycle refers to the usage of the outlet water stream from unit $j$ in the same unit $j$. It is worthy of note that absolute minimum wastewater generation can be achieved through a flexible rather than fixed schedule of the operations.

\section{Mathematical formulation}

The proposed mathematical formulation involves the following sets, variables and parameters. 


\subsection{Sets}

$P=\{p \mid p=$ time point $\}$

$J=\{j \mid j=$ unit $\}$

$C=\{c \mid c=$ contaminant $\}$

$S_{\text {in }}=\left\{S_{\text {in }} \mid S_{\text {in }}=\right.$ input state into any unit $\}$

$S_{\text {out }}=\left\{s_{\text {out }} \mid S_{\text {out }}=\right.$ output state from any unit $\}$

$S=\{s \mid s=$ any state $\}=S_{\text {in }} \cup S_{\text {out }}$

$S_{i n, j}=\left\{s_{i n, j} \mid S_{i n, j}=\right.$ input state into unit $\left.j\right\} \subseteq S_{i n}$

$S_{i n, j}^{*}=\left\{S_{i n, j}^{*} \mid S_{i n, j}^{*}=\right.$ input state into unit $\left.j\right\} \subseteq S_{i n, j}$

$S_{\text {out }, j}=\left\{s_{\text {out }, j} \mid S_{\text {out }, j}=\right.$ output state from unit $\left.j\right\} \subseteq S_{\text {out }}$

\subsection{Variables associated with wastewater minimisation}
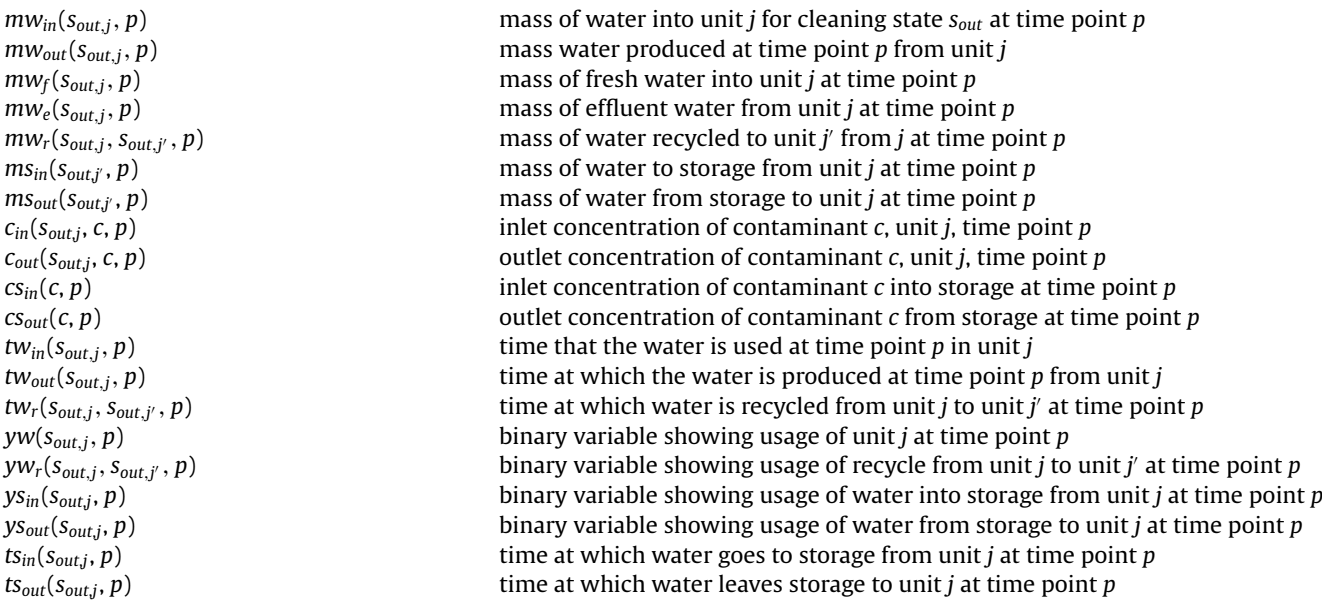

\subsection{Variables associated with production scheduling (Majozi \& Zhu, 2001)}

$\begin{array}{ll}t_{\text {out }}\left(s_{\text {out }, j}, p\right) & \text { time at which a state is produced from unit } j \text { at time point } p \\ t_{\text {in }}\left(s_{\text {out }, j}, p\right) & \text { time at which a state is used in or enters unit } j \text { at time point } p \\ q_{s}(s, p) & \text { amount of state } s \text { stored at time point } p \\ m_{\text {out }}\left(s_{\text {out }, j}, p\right) & \text { amount of state produced from unit } j \text { at time point } p \\ m_{\text {in }}\left(s_{\text {in }, j}, p\right) & \text { amount of state used in or enters unit } j \text { at time point } p \\ y\left(s_{\text {in }, j}^{*}, p\right) & \text { binary variable associated with usage of state } s \text { at time point } p \\ d\left(s_{\text {out }}, p\right) & \text { amount of state delivered to customers at time point } p\end{array}$

\subsection{Parameters associated with wastewater minimisation}

$\Psi(j)$
$S P(j)$
$C R(j)$
$C E$
$C F$
$M\left(s_{\text {out }, j}, c\right)$
$M w^{U}\left(s_{\text {out }, j}\right)$
$C_{\text {in }}^{U}\left(s_{\text {out }, j}, c\right)$
$C_{\text {out }}^{U}\left(s_{\text {out }, j}, c\right)$
$\tau w^{\prime}\left(s_{\text {out }, j}\right)$
$Q w_{s}^{o}$
$Q w_{s}^{U}$
$C S_{\text {out }}^{O}(c)$

\subsection{Parameters associated with production scheduling}

$\begin{array}{ll}V_{j}^{U} & \text { maximum design capacity of a particular unit } j \\ V_{j}^{L} & \text { minimum design capacity of a particular unit } j \\ H & \text { time horizon of interest } \\ \tau\left(s_{i n, j}^{*}\right) & \text { mean processing time for a state } \\ Q_{s}^{0}(s) & \text { initial amount of state } s \text { stored } \\ Q_{s}^{U}(s) & \text { maximum amount of state } s \text { stored within the time horizon of interest } \\ C P(s) & \text { selling price of product } s, s=\text { product }\end{array}$

\subsection{Wastewater minimisation without storage}

The mathematical formulation for the case where there is recycle and reuse of wastewater without intermediate storage is based on the superstructure given in Fig. 1 (Majozi, 2005). In each water using operation, water into the unit is a combination of the freshwater and water recycled from other units to that unit. Water leaving the unit can either be discarded as effluent or be recycled/reused in other 


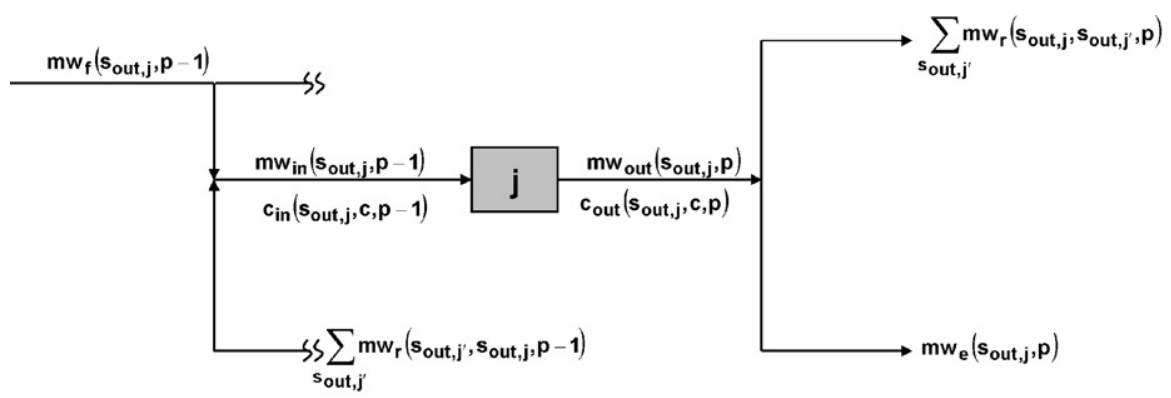

(a)

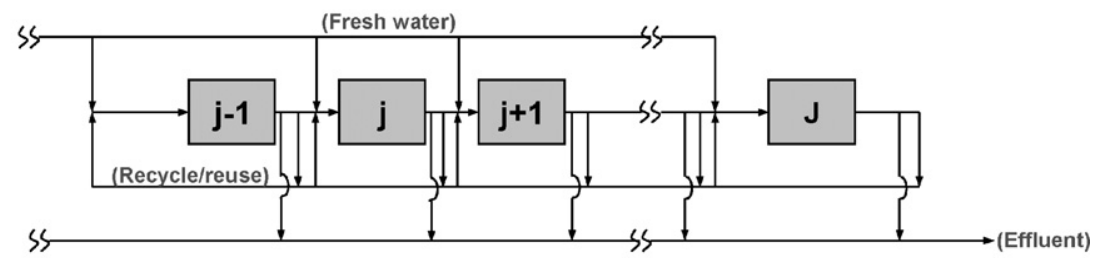

(b)

Fig. 1. Superstructure used for case with no storage.

units. It is highly imperative to realise that the water use and reuse variables shown in Fig. 1 correspond to the washing operation that follows immediately after the production of particular state in unit $j$, i.e. $s_{\text {out }, j}$. Consequently, these variables are task-specific, which allows scheduling to be readily embedded within the water reuse and recycle framework.

\subsection{Mass balance constraints without reusable water storage}

The first constraints considered are the mass balance constraints. A mass balance is done over the inlet into a unit $j$, over the outlet of the unit and over the unit itself. Constraint (1) is a water balance over the inlet to unit $j$. The total water into a unit is the sum of all the recycles to the unit and the fresh water into the unit $j$ at time point $p$. It is assumed that any operation that takes place in unit $j$ does not produce water as captured in constraint (2). Constraint (3) is an outlet water balance from unit $j$. Here the total water out of a unit is the sum of all the recycle streams to other units and the water discarded as effluent. Constraint (4) is a contaminant balance over unit $j$. It is also the definition if the outlet concentration. The mass of contaminants out of unit $j$ is the sum of the contaminants into the unit $j$ and the mass of contaminants added from the operation performed in the unit. As there are more than one contaminants present in the system the balance has to be done for each contaminant $c$. Constraint (5) is the definition of the inlet concentration of contaminant $c$ to unit $j$. The inlet concentration is defined as the mass of contaminants from the recycle streams divided by the total water into unit $j$ :

$$
\begin{aligned}
& m w_{\text {in }}\left(s_{\text {out }, j}, p\right)=\sum_{s_{\text {out } j^{\prime}}} m w_{r}\left(s_{\text {out }, j^{\prime}}, s_{\text {out }, j}, p\right)+m w_{f}\left(s_{\text {out }, j}, p\right), \forall j, j^{\prime} \in J, s_{\text {out }, j} \in S_{\text {out }, j}, p \in P \\
& m w_{\text {in }}\left(s_{\text {out }, j}, p-1\right)=m w_{\text {out }}\left(s_{\text {out }, j}, p\right), \forall j \in J, s_{\text {out }, j} \in S_{\text {out }, j}, p \in P \\
& m w_{\text {out }}\left(s_{\text {out }, j}, p\right)=\sum_{s_{\text {out } j^{\prime}}} m w_{r}\left(s_{\text {out }, j}, s_{\text {out }, j^{\prime}}, p\right)+m w_{e}\left(s_{\text {out }, j}, p\right), \forall j, j^{\prime} \in J, s_{\text {out }, j} \in S_{\text {out }, j}, p \in P \\
& m w_{\text {out }}\left(s_{\text {out }, j}, p\right) c_{\text {out }}\left(s_{\text {out }, j}, c, p\right)=m w_{\text {in }}\left(s_{\text {out }, j}, p-1\right) c_{\text {in }}\left(s_{\text {out }, j}, c, p-1\right)+M\left(s_{\text {out }}, c\right) y w\left(s_{\text {out }, j}, p-1\right), \forall j \in J, s_{\text {out }, j} \in S_{\text {out }, j}, p \in P, p>p_{1}, c \in C
\end{aligned}
$$

$$
c_{\text {in }}\left(s_{\text {out }, j}, c, p\right)=\frac{\sum_{s_{\text {out }, j^{\prime}}} m w_{r}\left(s_{\text {out }, j^{\prime}}, s_{\text {out }, j}, p\right) c_{\text {out }}\left(s_{\text {out }, j^{\prime}}, c, p\right)}{m w_{\text {in }}\left(s_{\text {out }, j}, p\right)}, \forall j, j^{\prime} \in J, s_{\text {out }, j^{\prime}} \in S_{\text {out }, j}, p \in P, c \in C
$$

The outlet concentration of each contaminant $c$ in unit $j$ cannot exceed its maximum limit as stated in constraint (6). Constraint (7) ensures that the total water into a unit $j$ does not exceed the maximum allowable for the operation in unit $j$. Constraint $(8)$ restricts the mass of water recycled into the unit $j$ to the maximum allowable water for the operation in unit $j$. Constraint (9) stipulates that the inlet concentration for contaminant $c$ into unit $j$ cannot exceed its upper limit.

$$
\begin{aligned}
& c_{\text {out }}\left(s_{\text {out }, j}, c, p\right) \leq C_{\text {out }}^{U}\left(s_{\text {out }, j}, c\right) y w\left(s_{\text {out }, j}, p-1\right), \forall j \in J, s_{\text {out }, j} \in S_{\text {out }, j}, p \in P, p>p_{1}, c \in C \\
& m w_{\text {in }}\left(s_{\text {out }, j}, p\right) \leq M w^{U}\left(s_{\text {out }, j}\right) y w\left(s_{\text {out }, j}, p\right), \forall j \in J, s_{\text {out }, j} \in S_{\text {out }, j}, p \in P \\
& m w_{r}\left(s_{\text {out }, j^{\prime}}, s_{\text {out }, j}, p\right) \leq M w^{U}\left(s_{\text {out }, j}\right) y w_{r}\left(s_{\text {out }, j^{\prime}}, s_{\text {out }, j}, p\right), \forall j, j^{\prime} \in J, s_{\text {out }, j} \in S_{\text {out }, j}, p \in P
\end{aligned}
$$




$$
c_{\text {in }}\left(s_{\text {out }, j}, c, p\right) \leq C_{\text {in }}^{U}\left(s_{\text {out }, j}, c\right) y w\left(s_{\text {out }, j}, p\right), \forall j \in J, s_{\text {out }, j} \in S_{\text {out }, j}, p \in P, c \in C
$$

The maximum water quantity into a unit is represented by Eq. (10). It is important to note that for multi-contaminant wastewater the outlet concentrations of the individual components cannot all be set to the maximum, since the contaminants are not limiting simultaneously. The limiting contaminant(s) will always be at the maximum outlet concentration and the non-limiting contaminants will be below their respective maximum outlet concentrations:

$$
M w^{U}\left(s_{\text {out }, j}\right)=\max _{c \in C}\left\{\frac{M\left(s_{\text {out }, j}, c\right)}{C_{\text {out }}^{U}\left(s_{\text {out }, j}, c\right)-C_{\text {in }}^{U}\left(s_{\text {out }, j}, c\right)}\right\}, \forall j \in J, s_{\text {out }, j} \in S_{\text {out }, j}
$$

Due to the discontinuous nature of batch processes the mass balances are not enough to fully describe the system. Sequencing constraints need to capture the discontinuous nature of the operation.

\subsection{Water recycle/reuse sequencing constraints}

Scheduling of the recycle/reuse streams is important because of the discontinuous manner of the streams existence. Wastewater can only be recycled/reused if the unit producing the wastewater and the unit receiving the wastewater finish operating and begin operating at the same time, respectively. This basically means that wastewater can only be recycled/reused once it has been produced. Furthermore wastewater can only be recycled/reused if there is a unit that starts operating at the time when water is available. The recycle/reuse of wastewater to a unit is also subject to concentration constraints. The first constraint considered is constraint (11). This constraint stipulates that recycle/reuse between units can only take place when the unit receiving the wastewater is operating at that time point. The unit receiving the wastewater, however, does not need to receive wastewater to operate, i.e. it can operate independent of the wastewater recycle/reuse. Constraints (12) and (13) ensure that the time at which recycle/reuse of water takes place is the same time as that at which water is produced. Constraints (14) and (15) ensure that the starting time of the unit receiving water coincides with the time at which that water is recycled/reused. It is worthy to note that in the absence of recycle/reuse, constraints (12)-(15) become redundant:

$$
\begin{aligned}
& y w_{r}\left(s_{\text {out }, j}, s_{\text {out }, j^{\prime}}, p\right) \leq y w\left(s_{\text {out }, j^{\prime}}, p\right), \forall j, j^{\prime} \in J, s_{\text {out }, j} \in S_{\text {out }, j} p \in P \\
& t w_{r}\left(s_{\text {out }, j}, s_{\text {out }, j^{\prime}}, p\right) \leq t w_{\text {out }}\left(s_{\text {out }, j}, p\right)+H\left(1-y w_{r}\left(s_{\text {out }, j}, s_{\text {out }, j^{\prime}}, p\right)\right), \forall j, j^{\prime} \in J, s_{\text {out }, j} \in S_{\text {out }, j}, p \in P \\
& t w_{r}\left(s_{\text {out }, j}, s_{\text {out }, j^{\prime}}, p\right) \geq t w_{\text {out }}\left(s_{\text {out }, j}, p\right)-H\left(1-y w_{r}\left(s_{\text {out }, j}, s_{\text {out }, j^{\prime}}, p\right)\right), \forall j, j^{\prime} \in J, s_{\text {out }, j} \in S_{\text {out }, j}, p \in P \\
& t w_{r}\left(s_{\text {out }, j}, s_{\text {out }, j^{\prime}}, p\right) \leq t w_{\text {in }}\left(s_{\text {out }, j^{\prime}}, p\right)+H\left(1-y w_{r}\left(s_{\text {out }, j}, s_{\text {out }, j^{\prime}}, p\right)\right), \forall j, j^{\prime} \in J, s_{\text {out }, j} \in S_{\text {out }, j}, p \in P \\
& t w_{r}\left(s_{\text {out }, j}, s_{\text {out }, j^{\prime}}, p\right) \geq t w_{\text {in }}\left(s_{\text {out }, j}, p\right)-H\left(1-y w_{r}\left(s_{\text {out }, j}, s_{\text {out }, j^{\prime}}, p\right)\right), \forall j, j^{\prime} \in J, s_{\text {out }, j} \in S_{\text {out }, j}, p \in P
\end{aligned}
$$

\subsection{Scheduling constraints associated with water recycle/reuse}

Each water using operation in the time horizon has to be scheduled accordingly, within the overall framework of operation scheduling. This is captured in Eqs. (16)-(19). Constraints (16) and (17) ensure that unit $j$ is washed immediately after completing a task that produces $s_{\text {out }, j}$. Constraint (18) is a duration constraint, which defines the starting and ending times of a unit $j$ during the washing operation. Constraint (19) stipulates that the washing operation can only commence at time point $p$ if the task producing state $s_{\text {out }, j}$ was activated at the previous time point. This could be considered as a complementary constraint to constraints (16)-(18). Constraint (20) is optional in the presence of constraint (72).

$$
\begin{aligned}
& t w_{\text {in }}\left(s_{\text {out }, j}, p\right) \geq t_{p}\left(s_{\text {out }, j}, p\right)-H\left(1-y w\left(s_{\text {out }, j}, p\right)\right), \forall j \in J, s_{\text {out }, j} \in S_{\text {out }, j}, p \in P \\
& t w_{\text {in }}\left(s_{\text {out }, j}, p\right) \leq t_{p}\left(s_{\text {out }, j}, p\right)+H\left(1-y w\left(s_{\text {out }, j}, p\right)\right), \forall j \in J, s_{\text {out }, j} \in S_{\text {out }, j}, p \in P \\
& t w_{\text {out }}\left(s_{\text {out }, j}, p\right)=t w_{\text {in }}\left(s_{\text {out }, j}, p-1\right)+\tau w\left(s_{\text {out }, j}\right) y w\left(s_{\text {out }, j}, p-1\right), \forall j \in J, s_{\text {out }, j} \in S_{\text {out }, j}, s_{\text {in }} \in S_{\text {in }}, p \in P, p>p_{1} \\
& y w\left(s_{\text {out }, j}, p\right)=y\left(s_{\text {in }, j}^{*}, p-1\right), \forall j \in J, s_{\text {out }, j} \in S_{\text {out }, j}, s_{\text {in }, j}^{*} \in S_{\text {in }, j}^{*}, s_{\text {in }, j}^{*} \rightarrow s_{\text {out }, j}, p \in P, p>p_{1} \\
& \sum_{s_{\text {out }, j}} y w\left(s_{\text {out }, j}, p\right) \leq 1, \forall j \in J, s_{\text {out }, j} \in S_{\text {out }, j}, p \in P
\end{aligned}
$$

\subsection{Linearisation}

Constraints (4) and (5) contain bilinear terms. This makes the model non-linear. The terms can be linearised according to the linearisation of bilinear terms proposed by Quesada and Grossmann (1995). The linearisation is as follows.

Let:

$$
\begin{aligned}
& c_{\text {in }}\left(s_{\text {out }, j}, c, p\right) m w_{\text {in }}\left(s_{\text {out }, j}, p\right)=\Gamma_{1}\left(s_{\text {out }, j}, c, p\right) \\
& c_{\text {out }}\left(s_{\text {out }, j}, c, p\right) m w_{\text {out }}\left(s_{\text {out }, j}, p\right)=\Gamma_{2}\left(s_{\text {out }, j}, c, p\right) \\
& c_{\text {out }}\left(s_{\text {out }, j^{\prime}}, c, p\right) m w_{r}\left(s_{\text {out }, j^{\prime}}, s_{\text {out }, j}, p\right)=\Gamma_{3}\left(s_{\text {out }, j^{\prime}}, s_{\text {out }, j}, c, p\right)
\end{aligned}
$$


with each variable having the following bounds:

$$
\begin{aligned}
& 0 \leq c_{\text {in }}\left(s_{\text {out }, j}, c, p\right) \leq C_{\text {in }}^{U}\left(s_{\text {out }, j}, c\right) \\
& 0 \leq m w_{\text {in }}\left(s_{\text {out }, j}, p\right) \leq M w^{U}\left(s_{\text {out }, j}\right) \\
& 0 \leq c_{\text {out }}\left(s_{\text {out }, j}, c, p\right) \leq C_{\text {out }}^{U}\left(s_{\text {out }, j}, c\right) \\
& 0 \leq m w_{\text {out }}\left(s_{\text {out }, j}, p\right) \leq M w^{U}\left(s_{\text {out }, j}\right) \\
& 0 \leq m w_{r}\left(s_{\text {out }, j^{\prime}}, s_{\text {out }, j}, p\right) \leq M w^{U}\left(s_{\text {out }, j}\right),
\end{aligned}
$$

then the following constraints are true for $\Gamma_{1}$ :

$$
\begin{aligned}
& \Gamma_{1}\left(s_{\text {out }, j}, c, p\right) \geq 0, \forall j \in J, s_{\text {out }, j} \in S_{\text {out }, j}, p \in P, c \in C \\
& \Gamma_{1}\left(s_{\text {out }, j}, c, p\right) \geq M w^{U}\left(s_{\text {out }, j}\right) c_{\text {in }}\left(s_{\text {out }, j}, c, p\right)+C_{\text {in }}^{U}\left(s_{\text {out }, j}, c\right) m w_{\text {in }}\left(s_{\text {out }, j}, c, p\right)-M w^{U}\left(s_{\text {out }, j}\right) C_{\text {in }}^{U}\left(s_{\text {out }, j}, c\right), \forall j \in J, s_{\text {out }, j} \in S_{\text {out }, j}, p \in P, c \in C \\
& \Gamma_{1}\left(s_{\text {out }, j}, c, p\right) \leq M w^{U}\left(s_{\text {out }, j}\right) c_{\text {in }}\left(s_{\text {out }, j}, c, p\right), \forall j \in J, s_{\text {out }, j} \in S_{\text {out }, j}, p \in P, c \in C \\
& \Gamma_{1}\left(s_{\text {out }, j}, c, p\right) \leq m w_{\text {in }}\left(s_{\text {out }, j}, p\right) C_{\text {in }}^{U}\left(s_{\text {out }, j}, c\right), \forall j \in J, s_{\text {out }, j} \in S_{\text {out }, j}, p \in P, c \in C
\end{aligned}
$$

and the following constraints for $\Gamma_{2}$ :

$$
\begin{aligned}
& \Gamma_{2}\left(s_{\text {out }, j}, c, p\right) \geq 0, \forall j \in J, s_{\text {out }, j} \in S_{\text {out }, j}, p \in P, c \in C \\
& \Gamma_{2}\left(s_{\text {out }, j}, c, p\right) \geq M w^{U}\left(s_{\text {out }, j}\right) c_{\text {out }}\left(s_{\text {out }, j}, c, p\right)+C_{\text {out }}^{U}\left(s_{\text {out }, j}, c\right) m w_{\text {out }}\left(s_{\text {out }, j}, c, p\right)-M w^{U}\left(s_{\text {out }, j}\right) C_{\text {out }}^{U}\left(s_{\text {out }, j}, c\right), \forall j \in J, s_{\text {out }, j} \in S_{\text {out }, j}, p \in P, c \in C
\end{aligned}
$$

$$
\Gamma_{2}\left(s_{\text {out }, j}, c, p\right) \leq m w_{\text {out }}\left(s_{\text {out }, j}, p\right) C_{\text {out }}^{U}\left(s_{\text {out }, j}, c\right), \forall j \in J, s_{\text {out }, j} \in S_{\text {out }, j}, p \in P, c \in C
$$

and the following constraints for $\Gamma_{3}$ :

$$
\begin{aligned}
\Gamma_{3}\left(s_{\text {out }, j^{\prime}}, s_{\text {out }, j}, c, p\right) \geq & 0, \forall j, j^{\prime} \in J, s_{\text {out }, j} \in S_{\text {out }, j}, p \in P, c \in C \\
\Gamma_{3}\left(s_{\text {out }, j^{\prime}}, s_{\text {out }, j}, c, p\right) \geq & M w^{U}\left(s_{\text {out }, j}\right) c_{\text {out }}\left(s_{\text {out }, j^{\prime}}, c, p\right)+C_{\text {out }}^{U}\left(s_{\text {out }, j^{\prime}}, c\right) m w_{r}\left(s_{\text {out }, j^{\prime}}, s_{\text {out }, j}, p\right) \\
& -M w^{U}\left(s_{\text {out }, j}\right) C_{\text {out }}^{U}\left(s_{\text {out }, j^{\prime}}, c\right), \forall j, j^{\prime} \in J, s_{\text {out }, j} \in S_{\text {out }, j}, p \in P, c \in C \\
\Gamma_{3}\left(s_{\text {out }, j^{\prime}}, s_{\text {out }, j}, c, p\right) \leq & M w^{U}\left(s_{\text {out }, j}\right) c_{\text {out }}\left(s_{\text {out }, j^{\prime}}, c, p\right), \forall j, j^{\prime} \in J, s_{\text {out }, j} \in S_{\text {out }, j} p \in P, c \in C \\
\Gamma_{3}\left(s_{\text {out }, j^{\prime}}, s_{\text {out }, j}, c, p\right) \leq & m w_{r}\left(s_{\text {out }, j^{\prime}}, s_{\text {out }, j}, p\right) C_{\text {out }}^{U}\left(s_{\text {out }, j^{\prime}}, c\right), \forall j, j^{\prime} \in J, s_{\text {out }, j} \in S_{\text {out }, j}, p \in P, c \in C
\end{aligned}
$$

Substituting the above linearised variables into constrains (4) and (5) give constraints (32) and (33):

$$
\begin{aligned}
& \Gamma_{2}\left(s_{\text {out }, j}, c, p\right)=\Gamma_{1}\left(s_{\text {out }, j}, c, p-1\right)+M\left(s_{\text {out }, j}, c\right) y w\left(s_{\text {out }, j}, p-1\right), \forall j \in J, s_{\text {out }, j} \in S_{\text {out }, j}, p \in P, c \in C \\
& \Gamma_{1}\left(s_{\text {out }, j}, c, p\right)=\sum_{s_{\text {out }, j^{\prime}}} \Gamma_{3}\left(s_{\text {out }, j^{\prime}}, s_{\text {out }, j}, c, p\right), \forall j, j^{\prime} \in J, s_{\text {out }, j} \in S_{\text {out }, j}, p \in P, c \in C
\end{aligned}
$$

\subsection{Wastewater minimisation with central storage}

The formulation of the model with storage is based on the superstructure given in Fig. 2. The total water flowing into the unit in this instance is the sum of the fresh water, the water recycled/reused from the other units and the water from storage. The water leaving the unit is then the sum of the water going to effluent, the water going to storage and the water being reused/recycled.

\subsection{Mass balance constraints with reusable water storage}

Constraints (1), (3) and (5) need to be modified to cater for the storage of the wastewater, thus yielding constraints (34), (35) and (36). The difference in the new constraint is that water into the unit is not only water from recycle and fresh water, but water from storage as well, given in constraint (34). This is similar for constraint (35) where outlet water involves recycled water, water that goes to effluent and water that goes to storage. The inlet concentration is not only affected by the contaminants from the recycle as in the previous case, but also the contaminants in the water from the storage tank as stated in constraint (36):

$$
\begin{aligned}
& m w_{\text {in }}\left(s_{\text {out }, j}, p\right)=\sum_{s_{\text {out }, j^{\prime}}} m w_{r}\left(s_{\text {out }, j^{\prime}}, s_{\text {out }, j}, p\right)+m w_{f}\left(s_{\text {out }, j}, p\right)+m s_{\text {out }}\left(s_{\text {out }, j}, p\right), \forall j, j^{\prime} \in J, s_{\text {out }, j} \in S_{\text {out }, j}, p \in P \\
& m w_{\text {out }}\left(s_{\text {out }, j}, p\right)=\sum_{s_{\text {out }, j^{\prime}}} m w_{r}\left(s_{\text {out }, j}, s_{\text {out }, j^{\prime}}, p\right)+m w_{e}\left(s_{\text {out }, j}, p\right)+m s_{\text {in }}\left(s_{\text {out }, j}, p\right), \forall j, j^{\prime} \in J, s_{\text {out }, j} \in S_{\text {out }, j}, p \in P
\end{aligned}
$$




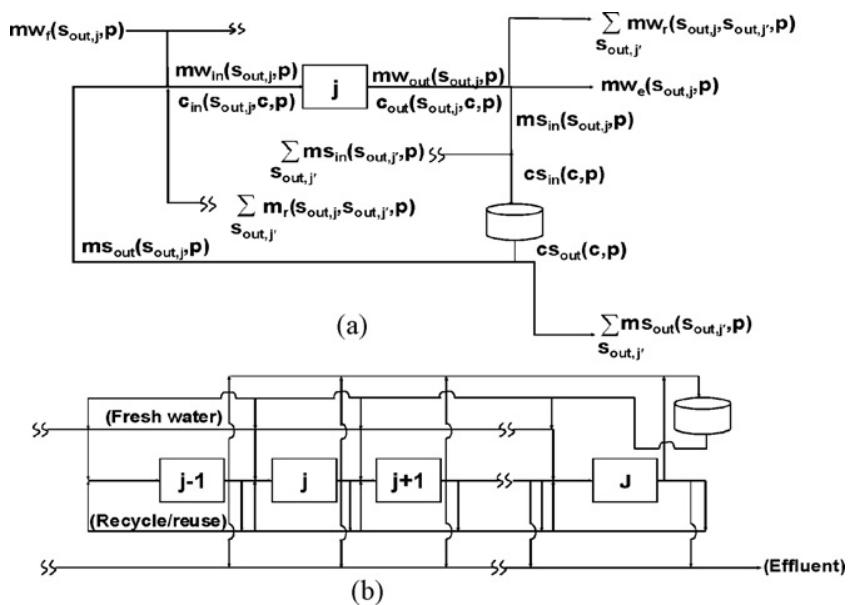

Fig. 2. Superstructure for model with storage.

$$
m w_{\text {in }}\left(s_{\text {out }, j}, p\right) c_{\text {in }}\left(s_{\text {out }, j}, c, p\right)=\sum_{s_{\text {out }, j^{\prime}}} m w_{r}\left(s_{\text {out }, j^{\prime}}, s_{\text {out }, j}, p\right) c_{\text {out }}\left(s_{\text {out }, j^{\prime}}, c, p\right)+m s_{\text {out }}\left(s_{\text {out }, j}, p\right) c s_{\text {out }}(c, p), \forall j, j^{\prime} \in J, s_{\text {out }, j^{\prime}} \in S_{\text {out }, j}, p \in P, c \in C
$$

One would notice that Eq. (36) contains terms that have been linearised previously as well as an additional non-linear term. The additional non-linear term is linearised in the similar manner as the other terms by noting that the maximum value of the mass flow is the maximum allowable water for the receiving unit and the maximum concentration of a contaminant in the storage vessel is the absolute maximum outlet concentration of that contaminant from all units.

Besides the mass balances over the water using operation, there also has to be mass balances over the storage unit. Constraint (37) is a water balance over the storage unit. The water in the storage vessel at a certain time point is the difference between the water flowing into and from the storage vessel and the water possibly stored from a previous time point. Constraint (38) is a water mass balance at the beginning of the time horizon. At the beginning of the time horizon there is no water flowing into the vessel, but there might be an initial amount of water in the vessel and hence there might be water leaving the vessel. Constraint (39) ensures that the water in the storage vessel does not exceed the storage vessels capacity. Constraint (40) is the definition of the inlet concentration into the storage vessel and constraint (41) is the definition of the outlet concentration from the vessel. It should be noted that constraint (41) is based on the assumption that the contaminant concentration inside the storage unit is the same as the concentration of the exit stream. Constraint (42) is the initial concentration of water in the storage tank at the beginning of the time horizon, provided there is an initial amount of water in the storage tank. Constraint (43) ensures that water flowing from storage to unit $j$ does not exceed unit $j$ 's capacity:

$$
\begin{aligned}
& q w_{s}(p)=q w_{s}(p-1)+\sum_{s_{\text {out }, j}} m s_{\text {in }}\left(s_{\text {out }, j}, p\right)-\sum_{s_{\text {out }, j}} m s_{\text {out }}\left(s_{\text {out }, j}, p\right), \forall j \in J, s_{\text {out }, j} \in S_{\text {out }, j}, p \in P, p>p_{1} \\
& q w_{s}\left(p_{1}\right)=Q w_{s}^{0}-\sum_{s_{\text {out }, j}} m s_{\text {out }}\left(s_{\text {out }, j}, p_{1}\right), \forall j \in J, s_{\text {out }, j} \in S_{\text {out }, j} \\
& q w_{s}(p) \leq Q w_{s}^{U}, \forall p \in P \\
& c s_{\text {in }}(c, p)=\frac{\sum_{s_{\text {out }, j}}\left(m s_{\text {in }}\left(s_{\text {out }, j}, p\right) c_{\text {out }}\left(s_{\text {out }, j}, c, p\right)\right)}{\sum_{s_{\text {out }, j}} m s_{\text {in }}\left(s_{\text {out }, j}, p\right)}, \forall j \in J, s_{\text {out }, j} \in S_{\text {out }, j}, p \in P, c \in C \\
& s_{\text {out }}(c, p)=\frac{q w_{s}(p-1) c s_{\text {out }}(c, p-1)+\left(\sum_{s_{\text {out }, j}} m s_{\text {in }}\left(s_{\text {out }, j}, p\right)\right) c s_{\text {in }}(c, p)}{q w_{s}(p-1)+\sum_{s_{\text {out }, j}} m s_{\text {in }}\left(s_{\text {out }, j}, p\right)}, \forall j \in J, s_{\text {out }, j} \in S_{\text {out }, j}, p \in P, p>p_{1}, c \in C \\
& c s_{\text {out }}\left(c, p_{1}\right)=C S_{\text {out }}^{0}(c), \forall c \in C \\
& m s_{\text {out }}\left(s_{\text {out }, j}, p\right) \leq M w^{U}\left(s_{\text {out }, j}\right) y s_{\text {out }}\left(s_{\text {out }, j}, p\right), \forall j \in J, s_{\text {out }, j} \in S_{\text {out }, j}, p \in P
\end{aligned}
$$

As one would notice there are non-linear terms in Eqs. (40) and (41). One of the non-linear terms can be negated by substituting Eq. (40) into Eq. (41). The non-linear terms in the resulting equation are then linearised in a similar fashion to that previously discussed.

\subsection{Sequencing constraints associated with storage}

Constraints (44) and (45) ensure that the time at which wastewater is produced from a unit is the same time at which the wastewater is transferred to storage. Furthermore, wastewater can only be sent to storage if unit $j$, which produced the wastewater, has operated. 
However, the fact that unit $j$ has operated does not mean that there is water that needs to be sent to storage from unit $j$. This is captured in constraint (46):

$$
\begin{aligned}
& t s_{\text {in }}\left(s_{\text {out }, j}, p\right) \geq t w_{\text {out }}\left(s_{\text {out }, j}, p\right)-H\left(1-y s_{\text {in }}\left(s_{\text {out }, j}, p\right)\right), \forall j \in J, s_{\text {out }, j} \in S_{\text {out }, j}, p \in P, p>p_{1} \\
& t s_{\text {in }}\left(s_{\text {out }, j}, p\right) \leq t w_{\text {out }}\left(s_{\text {out }, j}, p\right)+H\left(1-y s_{\text {in }}\left(s_{\text {out }, j}, p\right)\right), \forall j \in J, s_{\text {out }, j} \in S_{\text {out }, j}, p \in P, p>p_{1} \\
& y s_{\text {in }}\left(s_{\text {out }, j}, p\right) \leq y w\left(s_{\text {out }, j}, p-1\right), \forall j \in J, s_{\text {out }, j} \in S_{\text {out }, j}, p \in P, p>p_{1}
\end{aligned}
$$

Constraints (47) and (48) ensure that the time at which wastewater goes from storage to a unit $j$ is the same time at which unit $j$ starts its operation. Constraint (49) ensures that the unit is indeed operating when the unit is using wastewater from storage. The unit, however, does not necessarily have to use water from storage when it operates. A unit will not use the water in storage if there is a violation of the inlet concentration of the water into the unit or if there is simply no water in the storage vessel.

$$
\begin{aligned}
& t s_{\text {out }}\left(s_{\text {out }, j}, p\right) \geq t w_{\text {in }}\left(s_{\text {in }, j}, p\right)-H\left(1-y s_{\text {out }}\left(s_{\text {out }, j}, p\right)\right), \forall j \in J, s_{\text {out }, j} \in S_{\text {out }, j}, p \in P \\
& t s_{\text {out }}\left(s_{\text {out }, j}, p\right) \leq t w_{\text {in }}\left(s_{\text {in }, j}, p\right)+H\left(1-y s_{\text {out }}\left(s_{\text {out }, j}, p\right)\right), \forall j \in J, s_{\text {out }, j} \in S_{\text {out }, j}, p \in P \\
& y s_{\text {out }}\left(s_{\text {out }, j}, p\right) \leq y w\left(s_{\text {out }, j}, p\right), \forall j \in J, s_{\text {out }, j} \in S_{\text {out }, j}, p \in P
\end{aligned}
$$

Constraint (50) ensures that when water is transferred to a unit at time point $p$, the time at which this happens is later in the time horizon than any previous time water was transferred to other units at previous time points. Constraints (51) and (52) ensure that the time at which wastewater is moved from storage to different units at the same time point is the same time, i.e. all the water leaving the storage tank at time point $p$ leaves at the same time:

$$
\begin{aligned}
& t s_{\text {out }}\left(s_{\text {out }, j}, p\right) \geq t s_{\text {out }}\left(s_{\text {out }, j^{\prime}}, p^{\prime}\right)-H\left(2-y s_{\text {out }}\left(s_{\text {out }, j}, p\right)-y s_{\text {out }}\left(s_{\text {out }, j^{\prime}}, p^{\prime}\right)\right), \forall j, j^{\prime} \in J, s_{\text {out }, j} \in S_{\text {out }, j}, p, p^{\prime} \in P, p \geq p^{\prime} \\
& t s_{\text {out }}\left(s_{\text {out }, j}, p\right) \geq t s_{\text {out }}\left(s_{\text {out }, j^{\prime}}, p\right)-H\left(2-y s_{\text {out }}\left(s_{\text {out }, j}, p\right)-y s_{\text {out }}\left(s_{\text {out }, j^{\prime}}, p\right)\right), \forall j, j^{\prime} \in J, s_{\text {out }, j} \in S_{\text {out }, j}, p \in P \\
& t s_{\text {out }}\left(s_{\text {out }, j}, p\right) \leq t s_{\text {out }}\left(s_{\text {out }, j^{\prime}}, p\right)+H\left(2-y s_{\text {out }}\left(s_{\text {out }, j}, p\right)-y s_{\text {out }}\left(s_{\text {out }, j^{\prime}}, p\right)\right), \forall j, j^{\prime} \in J, s_{\text {out }, j} \in S_{\text {out }, j}, p \in P
\end{aligned}
$$

Constraint (53) is similar to constraint (50), but it applies to inlet streams to the storage tank. Constraints (54) and (55) ensure that the time at which there is wastewater going to storage from unit $j$ and unit $j^{\prime}$ corresponds to the same time at a time point:

$$
\begin{aligned}
& t s_{\text {in }}\left(s_{\text {out }, j}, p\right) \geq t s_{\text {in }}\left(s_{\text {out }, j^{\prime}}, p^{\prime}\right)-H\left(2-y s_{\text {in }}\left(s_{\text {out }, j}, p\right)-y s_{\text {in }}\left(s_{\text {out }, j^{\prime}}, p^{\prime}\right)\right), \forall j, j^{\prime} \in J, s_{\text {out }, j} \in S_{\text {out }, j}, p, p^{\prime} \in P, p \geq p^{\prime} \\
& t s_{\text {in }}\left(s_{\text {out }, j}, p\right) \geq t s_{\text {in }}\left(s_{\text {out }, j^{\prime}}, p\right)-H\left(2-y s_{\text {in }}\left(s_{\text {out }, j}, p\right)-y s_{\text {in }}\left(s_{\text {out }, j^{\prime}}, p\right)\right), \forall j, j^{\prime} \in J, s_{\text {out }, j} \in S_{\text {out }, j}, p \in P \\
& t s_{\text {in }}\left(s_{\text {out }, j}, p\right) \leq t s_{\text {in }}\left(s_{\text {out }, j^{\prime}}, p\right)+H\left(2-y s_{\text {in }}\left(s_{\text {out }, j}, p\right)-y s_{\text {in }}\left(s_{\text {out }, j^{\prime}}, p\right)\right), \forall j, j^{\prime} \in J, s_{\text {out }, j} \in S_{\text {out }, j}, p \in P
\end{aligned}
$$

Constraint (56) ensures that the outlet time of water from storage tank at a time point occurs later than the inlet time at previous time points. Constraints (57) and (58) ensure that at time point $p$ the time at which water is moved to storage is the same as the time at which water leaves the storage.

$$
\begin{aligned}
& t s_{\text {out }}\left(s_{\text {out }, j}, p\right) \geq t s_{\text {in }}\left(s_{\text {out }, j^{\prime}}, p^{\prime}\right)-H\left(2-y s_{\text {out }}\left(s_{\text {out }, j}, p\right)-y s_{\text {in }}\left(s_{\text {out }, j^{\prime}}, p^{\prime}\right)\right), \forall j, j^{\prime} \in J, s_{\text {out }, j} \in S_{\text {out }, j}, p, p^{\prime} \in P, p \geq p^{\prime} \\
& t s_{\text {out }}\left(s_{\text {out }, j}, p\right) \geq t s_{\text {in }}\left(s_{\text {out }, j^{\prime}}, p\right)-H\left(2-y s_{\text {out }}\left(s_{\text {out }, j}, p\right)-y s_{\text {in }}\left(s_{\text {out }, j^{\prime}}, p\right)\right), \forall j, j^{\prime} \in J, s_{\text {out }, j} \in S_{\text {out }, j}, p \in P \\
& t s_{\text {out }}\left(s_{\text {out }, j}, p\right) \leq t s_{\text {in }}\left(s_{\text {out }, j^{\prime}}, p\right)+H\left(2-y s_{\text {out }}\left(s_{\text {out }, j}, p\right)-y s_{\text {in }}\left(s_{\text {out }, j^{\prime}}, p\right)\right), \forall j, j^{\prime} \in J, s_{\text {out }, j} \in S_{\text {out }, j}, p \in P
\end{aligned}
$$

The following constraints ensure that all the water reuse and recycle opportunities are explored within the time horizon of interest:

$$
\begin{aligned}
& t s_{\text {in }}\left(s_{\text {out }, j}, p\right) \leq H, \quad \forall j \in J, s_{\text {out }, j} \in S_{\text {out }, j}, p \in P \\
& t s_{\text {out }}\left(s_{\text {out }, j}, p\right) \leq H, \quad \forall j \in J, s_{\text {out }, j} \in S_{\text {out }, j}, p \in P \\
& t w_{\text {in }}\left(s_{\text {out }, j}, p\right) \leq H, \quad \forall j \in J, s_{\text {out }, j} \in S_{\text {out }, j}, p \in P \\
& t w_{\text {out }}\left(s_{\text {out }, j}, p\right) \leq H, \quad \forall j \in J, s_{\text {out }, j} \in S_{\text {out }, j}, p \in P \\
& t w_{r}\left(s_{\text {out }, j}, s_{\text {out }, j^{\prime}}, p\right) \leq H, \quad \forall j, j^{\prime} \in J, s_{\text {out }, j} \in S_{\text {out }, j}, p \in P
\end{aligned}
$$

\section{Production scheduling constraints}

The operation scheduling model has been presented in detail in another publication (Majozi \& Zhu, 2001), but is briefly presented in this section of the paper for continuity purposes.

\subsection{Capacity constraint} unit:

The capacity constraint ensures that the amount of material processed in unit $j$ at any time point $p$ does not exceed the capacity of the

$$
V_{j}^{L} y\left(s_{i n, j}^{*}, p\right) \leq \sum_{s_{i n, j}} m_{i n}\left(s_{i n, j}, p\right) \leq V_{j}^{U} y\left(s_{i n, j}^{*}, p\right), \forall j \in J, p \in P, s_{i n, j} \in S_{i n, j}, s_{i n, j}^{*} \in S_{i n, j}^{*}
$$




\subsection{Material balances}

The material balances ensure the conservation of mass around each unit and concerning each state involved in production scheduling:

$$
\begin{aligned}
& \sum_{s_{\text {in }, j}} m_{\text {in }}\left(s_{\text {in }, j}, p-1\right)=\sum_{s_{\text {out }, j}} m_{\text {out }}\left(s_{\text {out }, j}, p\right), \forall p \in P, p>p_{1}, j \in J, \forall s_{\text {in }, j} \in S_{\text {in }, j}, s_{\text {out }, j} \in S_{\text {out }, j} \\
& q_{s}\left(s, p_{1}\right)=Q_{S}^{0}(s)-m_{\text {in }}\left(s, p_{1}\right), s \neq \text { product, } \forall s \in S \\
& q_{s}(s, p)=q_{s}(s, p-1)-m_{\text {in }}(s, p), s=\text { feed, } \forall s \in S, \forall p \in P, p>p_{1} \\
& q_{s}(s, p)=q_{s}(s, p-1)+m_{\text {out }}(s, p)-m_{\text {in }}(s, p), s \neq \text { product, feed, } \forall s \in S, \forall p \in P, p>p_{1} \\
& q_{s}\left(s, p_{1}\right)=Q_{s}^{0}(s)-d\left(s, p_{1}\right), s=\text { product, } \forall s \in S \\
& q_{s}(s, p)=q(s, p-1)+m_{\text {out }}(s, p)-d(s, p), s=\text { product, byproduct, } \forall s \in S, \forall p \in P, p>p_{1}
\end{aligned}
$$

\subsection{Duration constraint}

The duration constraint constitutes one of the most crucial constraints as it addresses the intrinsic aspect of time in batch plants. It simply states that the time at which a particular state is produced is dependent on the duration of task that produces the same state as follows:

$$
t_{\text {out }}\left(s_{\text {out }, j}, p\right)=t_{i n}\left(s_{i n, j}^{*}, p-1\right)+\tau\left(s_{i n, j}^{*}\right) y\left(s_{i n, j}^{*}, p-1\right), \forall j \in J, p \in P, p>p_{1}, s_{i n, j}^{*} \in S_{i n, j}^{*}, s_{\text {out }, j} \in S_{\text {out }, j}
$$

\subsection{Assignment constraint}

The assignment constraint ensures that only one task takes place in a given unit at a given point in time:

$$
\sum_{s_{i n, j}^{*}} y\left(s_{i n, j}^{*}, p\right) \leq 1, \forall p \in P, j \in J, s_{i n, j}^{*} \in S_{i n, j}^{*}
$$

\subsection{Storage constraint}

The storage constraint is, in essence, the extension of the mass balances. It ensures that the amount of a particular state that is stored at any point in time during the time horizon of interest does not exceed the maximum allowed:

$$
q_{s}(s, p) \leq Q^{U}(s), \forall s \in S, p \in P
$$

\section{Sequence constraints for combined scheduling and wastewater minimisation}

In the presence of wastewater minimisation, the sequence constraints initially presented in Majozi and Zhu (2001) are modified as follows. The following constraint stipulates that the task corresponding to state $s_{i n, j}^{*}$ can only commence once all the previous tasks and their corresponding washing operations are complete:

$$
t_{\text {in }}\left(s_{\text {in }, j}, p\right) \geq \sum_{s_{\text {out }, j}}\left\{\left(\tau\left(s_{i n, j}^{*}\right)+\tau w\left(s_{\text {out }, j}\right)\right) y w\left(s_{\text {out }, j}, p^{\prime}-1\right)\right\}, \forall j \in J, p, p^{\prime} \in P, p \geq p^{\prime}, p^{\prime} \geq 2, s_{i n, j}^{*} \in S_{i n, j}^{*}, s_{i n, j} \in S_{\text {in }, j}, s_{\text {out }, j} \in S_{\text {out }, j}, s_{\text {in }, j}^{*} \rightarrow s_{\text {out }, j}
$$

\section{Solution procedure}

The solution procedure followed is similar to the method described by Gouws et al. (2008). The linearised form of the model is first solved to find an initial starting point for the non-linearised model. The non-linearised model is then solved. The solution found for the MILP is a relaxed solution, and therefore can, in certain instances, be infeasible. However, this is not a problem since the solution from the MILP only serves as the starting point for the MINLP and gives an upper bound (or lower bound depending on whether one is maximising profit or minimising effluent). Furthermore, if the solution to the MILP is feasible and the objective function value of the MILP and MINLP are the same, then the solution to the MINLP can be taken as the globally optimal solution. An illustrative example is provided to demonstrate the effectiveness of the model and the solution strategy.

\section{Illustrative example}

The illustrative example involves three water using vessels with three contaminants present. The data used in the example is given in Tables 1 and 2. The maximum water amounts given in Table 2 were calculated using Eq. (10).

The cost of treatment for effluent water is 200 c.u. A further specification given is the amount of water needed to produce the required product. For process $1,1 \mathrm{~kg}$ of water (aqueous phase) is needed for $3 \mathrm{~kg}$ of raw material. For process $2,1 \mathrm{~kg}$ of water is needed for $2 \mathrm{~kg}$ of raw material and for process $3,1 \mathrm{~kg}$ of water is needed for $1.5 \mathrm{~kg}$ of raw material. To calculate the mass of raw material used, the mass of water used is multiplied by the required factor given above. This is shown in Eq. (75). The mass of product is simply the mass of raw materials 
Table 1

Data for illustrative example.

\begin{tabular}{|c|c|c|c|c|}
\hline Process & Contaminant & $C_{i n}^{U}(j)(\mathrm{ppm})$ & $C_{\text {out }}^{U}(j)(\mathrm{ppm})$ & Mass load (g) \\
\hline \multirow{3}{*}{1} & 1 & 0 & 15 & 675 \\
\hline & 2 & 0 & 400 & 18,000 \\
\hline & 3 & 0 & 35 & 1,575 \\
\hline \multirow{3}{*}{2} & 1 & 20 & 120 & 3,400 \\
\hline & 2 & 300 & 12,500 & $4,14,800$ \\
\hline & 3 & 45 & 180 & 4,000 \\
\hline \multirow{3}{*}{3} & 1 & 120 & 220 & 5,600 \\
\hline & 2 & 20 & 45 & 1,400 \\
\hline & 3 & 200 & 9,500 & $5,20,800$ \\
\hline
\end{tabular}

Table 2

Further data for the illustrative example.

\begin{tabular}{|c|c|c|c|c|}
\hline Process & Duration (h) & Max. water (ton) & Selling price (c.u.) & Cost raw (c.u.) \\
\hline 1 & 2 & 45 & 2300 & 108 \\
\hline 2 & 2.5 & 34 & 2000 & 82 \\
\hline 3 & 1.5 & 56 & 1050 & 95 \\
\hline
\end{tabular}

entering a unit excluding the amount of material removed by water during the units operation. This is shown in Eq. (76). The objective function used is the maximisation of profit and is given in Eq. (77):

$$
\begin{aligned}
& \operatorname{mraw}\left(s_{\text {in }, j}, p\right)=m_{u}\left(s_{i n, j}, p\right) \Psi\left(s_{\text {in }}\right), \forall j \in J, s_{\text {in }} \in s_{\text {in }}, s_{\text {in }, j} \in S_{\text {in }, j}, p \in P \\
& \operatorname{mprod}\left(s_{\text {out }, j}, p\right)=\operatorname{mraw}\left(s_{\text {in }, j}, p-1\right)-\sum_{c} M\left(s_{\text {in }, j}, c\right) y\left(s_{\text {in }, j}, p-1\right), \forall j \in J, s_{\text {in }, j} \in S_{\text {in }, j}, s_{\text {out }, j} \in S_{\text {out }, j}, p \in P, c \in C \\
& R=\sum_{p}\left(\sum_{s_{\text {out }, j}} S P\left(s_{\text {out }}\right) \operatorname{mprod}\left(s_{\text {out }, j}, p\right)-\sum_{s_{i n, j}} C R\left(s_{\text {in }}\right) \operatorname{mraw}\left(s_{\text {in }, j}, p\right)-C E \sum_{s_{\text {in }, j}} m e\left(s_{\text {in }, j}, p\right)\right), \forall j \in J, s_{\text {out }} \in S_{\text {out }}, s_{\text {in }, j} \in S_{\text {in }, j}, s_{\text {out }, j} \in S_{\text {out }, j}, p \in P
\end{aligned}
$$

The illustrative example was solved for both cases presented in this paper, i.e. the case where there is no storage and the case where there is storage for wastewater.

\subsection{Wastewater minimisation without storage}

The example was solved using GAMS, with CPLEX for the linearised model and DICOPT for the non-linear model with CPLEX as the MIP solver and CONOPT as the NLP solver. The computer used had a Pentium 4, 3.2 GHz processor with 512 MB RAM. The final value of the objective function is $1.860 \times 10^{6} \mathrm{c}$.u. The final solution was found in $2.52 \mathrm{CPU}$ seconds with 72 binary variables. The total amount of effluent water produced is 552.73 tons. Had there been no recycle/reuse of wastewater the total effluent water would be 562 tons. This relates to a saving of $1.6 \%$. Fig. 3 shows a Gantt chart of the optimum schedule that achieves the minimum wastewater. The bold figures below the lines show the quantity of fresh water used by that unit. Whilst indeed optimal, the solution shown cannot be considered globally optimum since the objective function value for the linearised model did not equal that on the non-linear model. The value of the linear model is $1.8602 \times 10^{6}$ c.u. The main reason for the difference in the objective functions is due to the fact that the solution found for the

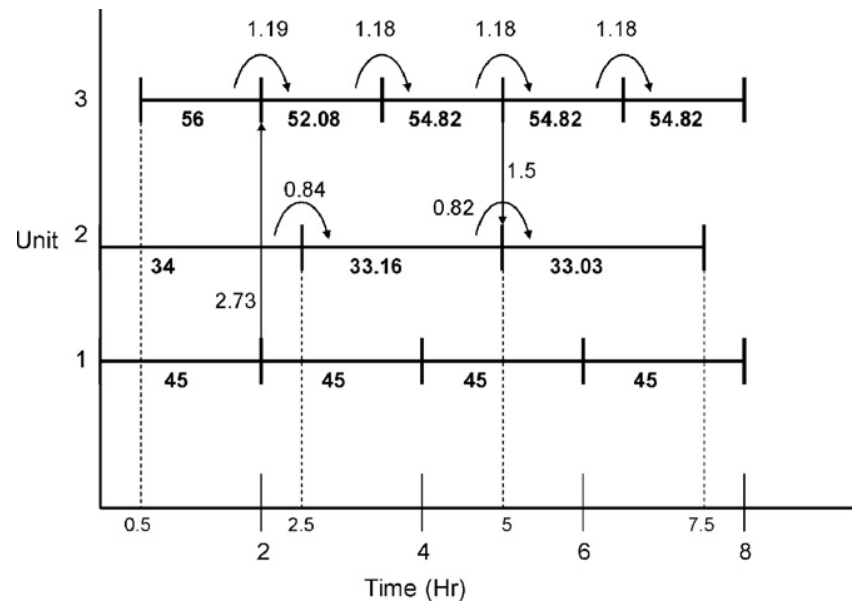

Fig. 3. Gantt chart for illustrative example with no storage. 


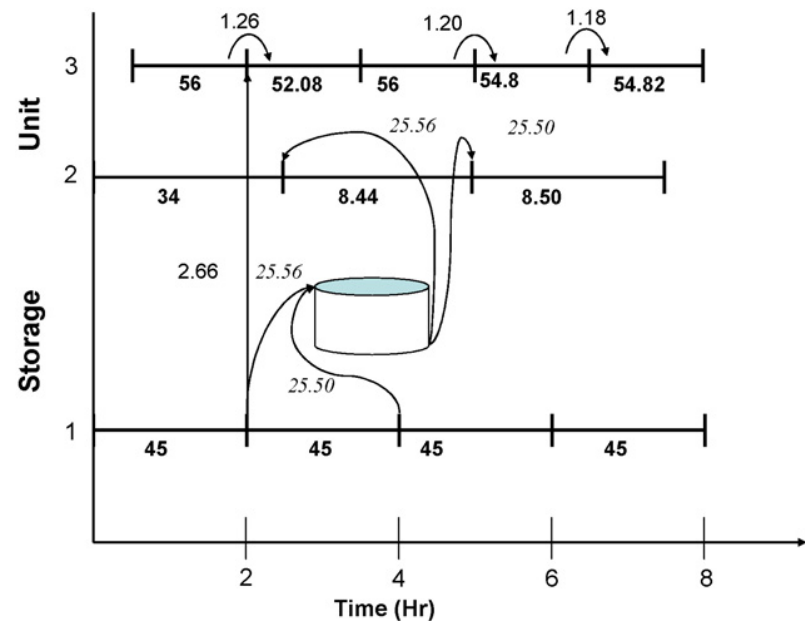

Fig. 4. Gantt chart for illustrative example with central storage.

MILP had infeasibilities in some of the mass balances. It is worthy of mention, however, that the issue of global optimality on time point based formulations like the one proposed, is subject to appropriate number of time points. This involves an iterative procedure in which the number of time points is incremented until there is no improvement in the objective function. In this case, convergence was achieved after 6 time points.

Fig. 3 shows that unit 3 is constantly recycling to itself. The same can be said for unit 2 . Unit 1 recycles to unit 3 after 2 h from the beginning of the time horizon. What is important to note is that the recycle/reuse amounts are restricted by multiple inlet concentration considerations. The reason why there has not been a large saving in wastewater is because the maximum inlet concentration of some of the contaminants is very low, which means that not much contaminated water can be recycled.

\subsection{Wastewater minimisation with central storage}

The illustrative example was also solved for the case where there is central reusable water storage. The maximum storage available for the wastewater is 200 tons. Important to note is that to truly minimise wastewater with storage the storage vessel should be empty at the end of the time horizon. Constraint (78) ensures that this is the case. Any water stored at the end of the time horizon would serve no purpose except to give a false savings in water used:

$$
q(p)=0, \quad p=|P|
$$

The computer used to solve the model had a Pentium 4, $3.2 \mathrm{GHz}$ processor with $512 \mathrm{MB}$ RAM. The problem was solved using GAMS with CPLEX as the MILP solver and DICOPT for the MINLP with CPLEX as the MIP solver and CONOPT as the NLP solver. The total solution time to the problem was $522.07 \mathrm{CPU}$ seconds with 162 binary variables. The value of the objective function is $1.869 \times 10^{6}$ c.u. using 9 time points. The total amount of effluent water generated is 504.63 tons. Had there been no reuse or recycle of the wastewater the total effluent would be 562 tons. The solution achieved a 10.2\% decrease in the total effluent water. Fig. 4 shows the Gantt chart for the solution.

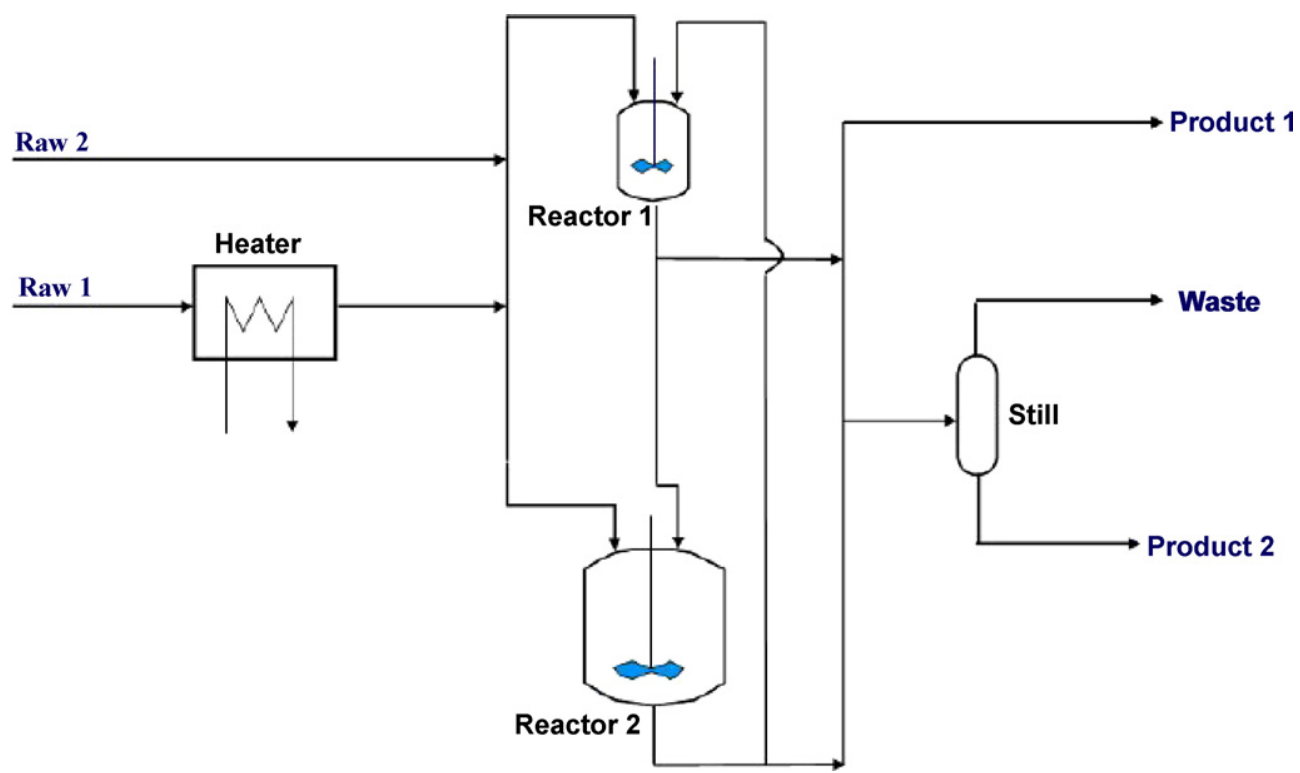

Fig. 5. Flowsheet for BATCH1 multipurpose facility. 

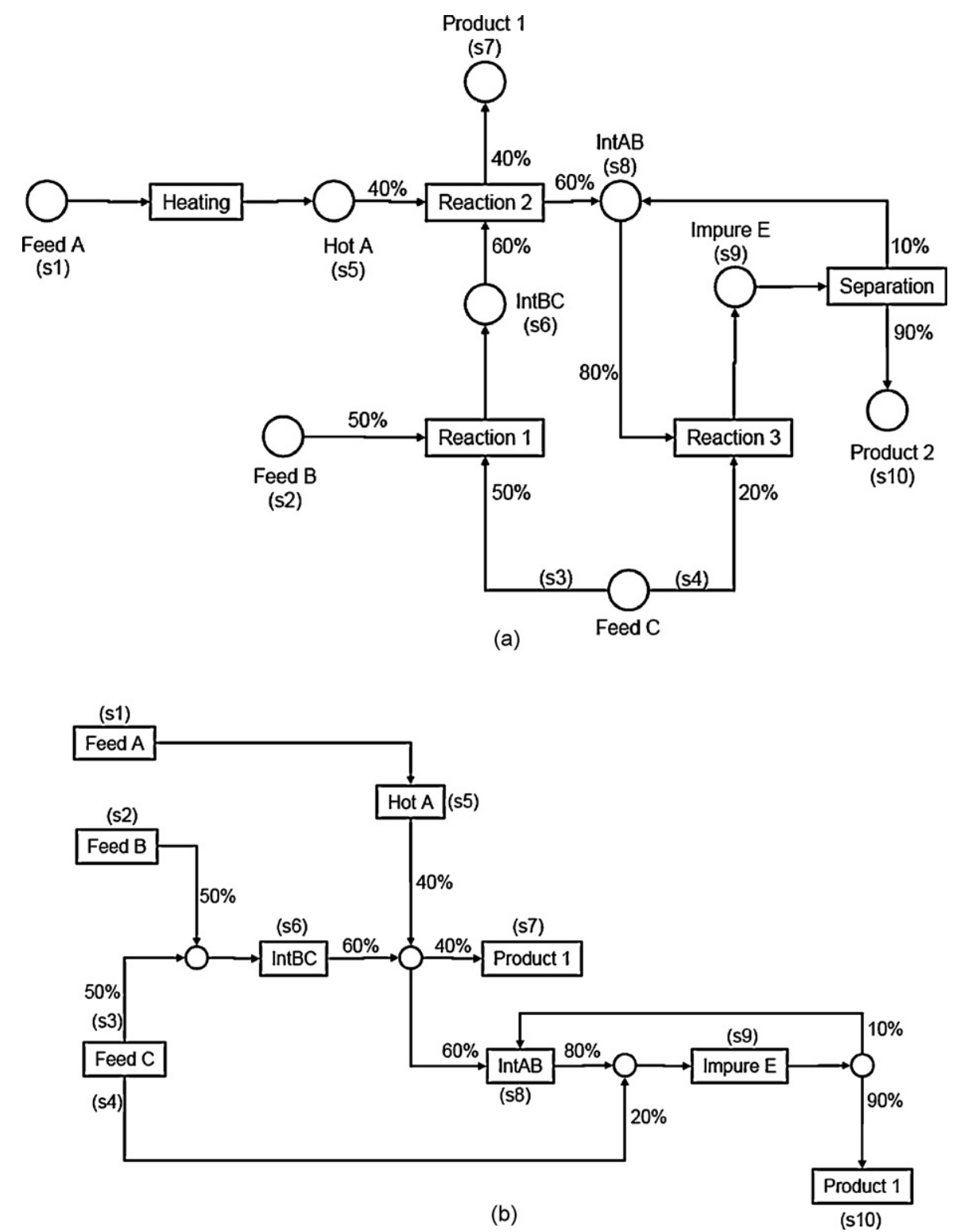

Fig. 6. (a) STN and (b) SSN for the literature example.

The solution to the MILP in this case, as with the previous case, was infeasible. The value of the objective function used as the starting point for the MINLP was $2.07 \times 10^{6}$ c.u. Furthermore, this solution was not an optimal solution to the MILP, but it had a relative gap of $9.8 \%$. When the MILP solution is forced to have a zero relative gap the solution times became excessive and the resulting solutions obtained from the MINLP were considerable lower in quality to that achieved by using the MILP solution with the higher relative gap. The only possible reason for this is that the solution provided by the MILP with a low relative gap starts the MINLP in a solution space dominated by a lower local optimum. It must also be noted that the solution obtained with a relative gap of zero was infeasible. This means that the usage of the higher relative gap solution is permitted. Had the zero relative gap solution to the MILP been feasible, then obviously one would have to use this solution as the starting point. Appropriate to emphasize, however, is the fact that the MINLP solution is indeed an optimal solution, albeit local.

As expected, the total water used in the case where there is storage is less than the case where there is no storage. The relative values of the maximum inlet concentrations will affect the amount of wastewater that can be reused or recycled. Had one of the maximum concentrations been zero for a particular contaminant for each unit there would have been no reuse or recycle of wastewater. What is also important to note is that the objective function not only minimises the wastewater but it also maximises profit. This means that to achieve the highest profit all the units will operate as many times as possible in a time horizon.

\section{Literature example (Kondili, Pantelides, \& Sargent, 1993)}

The case study involves a well-published multipurpose facility which is commonly known as BATCH1 in literature. It mainly consists of three chemical reactions which take place in two common reactors. In addition to the two common reactors, the flowsheet also entails the heater and the separator, before and after the reactors, respectively, as shown if Fig. 5. The STN and SSN for the literature example are given in Fig. 6(a) and (b), respectively. The data for this example appears in Table 3. 
Table 3

Scheduling data for the literature example.

\begin{tabular}{|c|c|c|c|}
\hline Unit & Capacity & Suitability & Mean processing time $(\tau)$ \\
\hline Heater $(j=1)$ & 100 & Heating & 1.0 \\
\hline Reactor $1(j=2)$ & 50 & Reaction $1,2,3$ & $2.0,2.0,1.0$ \\
\hline Reactor $2(j=3)$ & 80 & Reaction 1, 2, 3 & $2.0,2.0,1.0$ \\
\hline Still $(j=4)$ & 200 & Separation & 1 for product 2,2 for Int $A B$ \\
\hline State & Storage capacity & Initial amount & Price \\
\hline Feed A & Unlimited & Unlimited & 0.0 \\
\hline Feed B & Unlimited & Unlimited & 0.0 \\
\hline Feed C & Unlimited & Unlimited & 0.0 \\
\hline Hot A & 100 & 0.0 & 0.0 \\
\hline Int $A B$ & 200 & 0.0 & 0.0 \\
\hline IntBC & 150 & 0.0 & 0.0 \\
\hline Impure E & 200 & 0.0 & 0.0 \\
\hline Product 1 & Unlimited & 0.0 & 100.0 \\
\hline Product 2 & Unlimited & 0.0 & 100.0 \\
\hline
\end{tabular}

In order to illustrate the capability of the proposed technique, this multipurpose example has been enhanced by including compulsory washing operations after each of the reactions in each of the two reactors. The philosophy is that the reactors need to be cleaned after each reaction in order to remove contaminants that are formed as byproducts, so as to ensure product integrity. Data pertaining to cleaning tasks is shown in Table 4. The variation in performance in the two reactors could be ascribed to differences in design, which is indeed a common encounter in practice. In addition to this data, it is known that freshwater cost is 2 c.u./ $/ \mathrm{kg}$ of water whilst the effluent treatment cost is 3 c.u. $/ \mathrm{kg}$.

The objective function for the literature example is the maximization of a profit function over a $10 \mathrm{~h}$ time horizon that takes revenue, freshwater and wastewater treatment costs as follows:

$$
\operatorname{Max} Z=\sum_{s} \sum_{p} C P(s) d(s, p)-C F \sum_{s_{\text {out }, j}} \sum_{p} m w_{f}\left(s_{\text {out }, j}, p\right)-C E \sum_{s_{\text {out }, j}} \sum_{p} m w_{e}\left(s_{\text {out }, j}, p\right)
$$

Table 4

Wastewater minimisation data for the literature example.

\begin{tabular}{|c|c|c|c|c|}
\hline & & \multicolumn{3}{|c|}{ Maximum concentration ( $\mathrm{g}$ contaminant/kg water) } \\
\hline & & Contaminant 1 & Contaminant 2 & Contaminant 3 \\
\hline \multirow[t]{2}{*}{ Reaction 1 (Reactor 1 ) } & Max. inlet & 0.5 & 0.5 & 2.3 \\
\hline & Max. outlet & 1.0 & 0.9 & 3.0 \\
\hline \multirow[t]{2}{*}{ Reaction 2 (Reactor 1 ) } & Max. inlet & 0.01 & 0.05 & 0.3 \\
\hline & Max. outlet & 0.2 & 0.1 & 1.2 \\
\hline \multirow[t]{2}{*}{ Reaction 3 (Reactor 1 ) } & Max. inlet & 0.15 & 0.2 & 0.35 \\
\hline & Max. outlet & 0.3 & 1.0 & 1.2 \\
\hline \multirow[t]{2}{*}{ Reaction 1 (Reactor 2) } & Max. inlet & 0.05 & 0.2 & 0.05 \\
\hline & Max. outlet & 0.1 & 1 & 12 \\
\hline \multirow[t]{2}{*}{ Reaction 2 (Reactor 2) } & Max. inlet & 0.03 & 0.1 & 0.2 \\
\hline & Max. outlet & 0.075 & 0.2 & 1 \\
\hline \multirow[t]{4}{*}{ Reaction 3 (Reactor 2) } & Max. inlet & 0.3 & 0.6 & 1.5 \\
\hline & Max. outlet & 2.0 & 1.5 & 2.5 \\
\hline & & \multicolumn{3}{|l|}{ Mass load (g) } \\
\hline & & Contaminant 1 & Contaminant 2 & Contaminant 3 \\
\hline \multirow{2}{*}{ Reaction 1} & Reactor 1 & 4 & 80 & 10 \\
\hline & Reactor 2 & 15 & 24 & 358 \\
\hline \multirow{2}{*}{ Reaction 2} & Reactor 1 & 28.5 & 7.5 & 135 \\
\hline & Reactor 2 & 9 & 2 & 16 \\
\hline \multirow{4}{*}{ Reaction 3} & Reactor 1 & 15 & 80 & 85 \\
\hline & Reactor 2 & 22.5 & 45 & 36.5 \\
\hline & \multicolumn{3}{|c|}{ Duration of washing (h) } & \\
\hline & Reaction 1 & Reaction 2 & Reaction 3 & \\
\hline Reactor 1 & 0.25 & 0.50 & 0.25 & \\
\hline Reactor 2 & 0.30 & 0.25 & 0.25 & \\
\hline
\end{tabular}




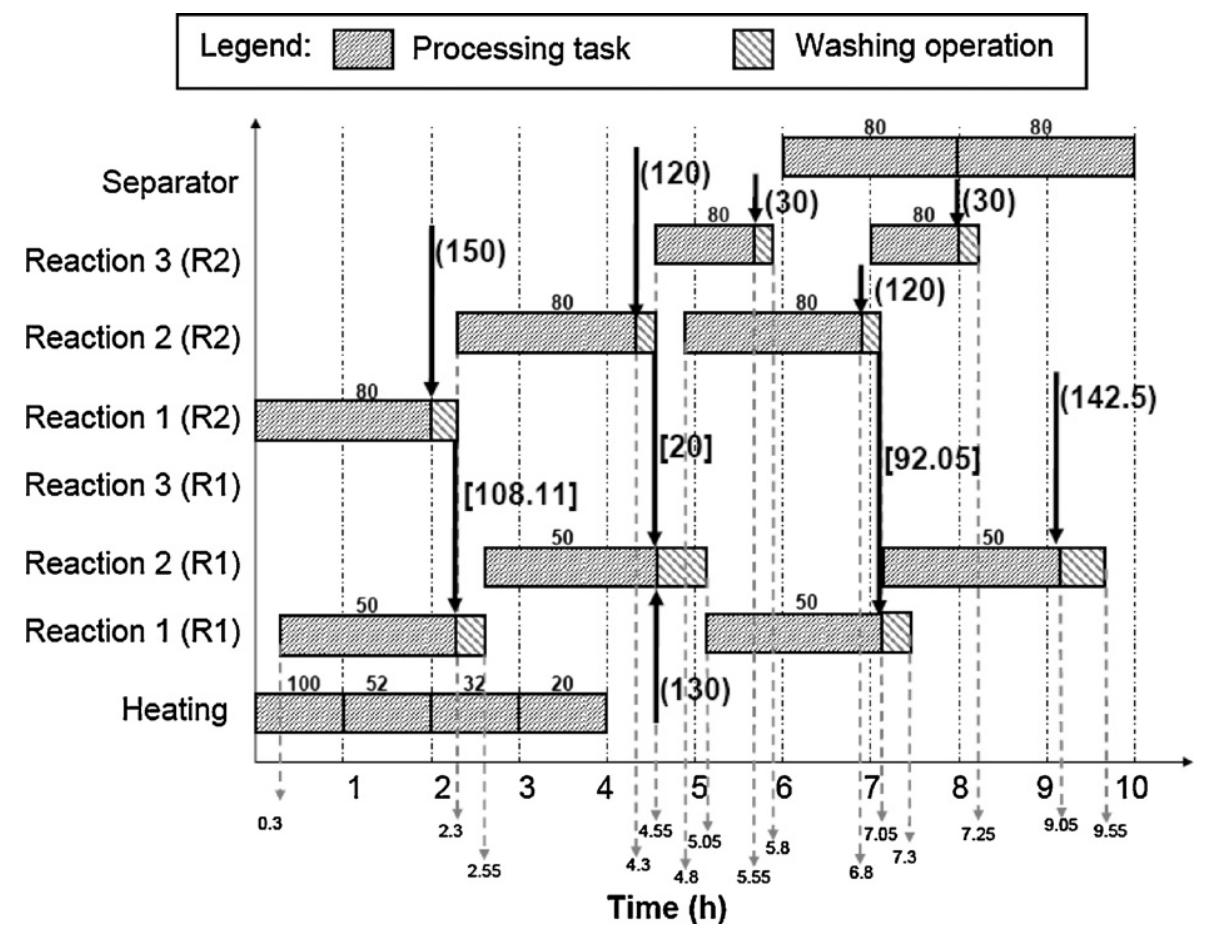

Fig. 7. Optimum schedule including recycle and reuse for BATCH1.

\subsection{Results for the literature example}

Fig. 7 shows the optimum production and wastewater minimisation schedule for the BATCH1 example. The values in curly brackets represent the amount of freshwater, in $\mathrm{kg}$, used at any given instant. On the other hand, the values in square brackets represent reused water from one operation to another. The values on top of the horizontal blocks represent the amount of material that is processed for production scheduling purposes. This schedule corresponds to an optimum objective value of 21187.5 c.u., which is at most $1.4 \%$ from the upper bound set by the relaxed problem. Consequently, this solution is guaranteed to be at most $1.4 \%$ from the global optimum. Concomitant with this solution is the usage of $722.5 \mathrm{~kg}$ of freshwater. In the absence of possible recycle and reuse, the optimum objective was found to be 20236.1 c.u., which is a proven global optimum due to its convergence with the reformulated relaxed objective. This corresponds to $912.8 \mathrm{~kg}$ of freshwater use. Therefore, in addition to improving the economic objective function, exploitation of recycle/reuse opportunities results in almost $21 \%$ reduction in freshwater use and effluent generation.

\section{Concluding remarks}

The methodology presented provides a means to minimise wastewater in batch processes where there are multiple contaminants present. Two cases are dealt with, namely the case where there is no storage for the wastewater and the case where there is finite storage of wastewater. Both cases are solved using a novel solution method presented by Gouws et al. (2008).

The proposed methodology does not require the schedule to be determined a priori as with other methodologies developed previously. The methodology rather determines the schedule that will generate the least amount of wastewater using recycle and reuse opportunities. To ensure that the least amount of wastewater is generated the schedule must be flexible to take advantage of this scenario. A further advantage of the schedule being flexible is that the methodology can be applied to any time horizon with relative ease and only the durations of the individual tasks needs to be specified.

The presented methodology finds broader application because it deals with wastewater with multiple contaminants, which is more prevalent in industry than wastewater with a single contaminant.

The model was applied to two example problems. In the first example, both direct and indirect reuse opportunities were considered. As is to be expected, the case where there was storage available for wastewater generated less wastewater than the case where there was no storage. The former had a $10.2 \%$ decrease in wastewater. Where there was no storage available for wastewater the water savings was only $1.6 \%$. In the more complex BATCH1 literature example, this methodology resulted in $20.8 \%$ savings in wastewater generation, whilst taking scheduling considerations into account. It is worthy of note that one cannot compare water savings in a single contaminant process to water savings where there are multiple contaminants, even though they share similar characteristics. The amount of wastewater that can be saved will be highly dependant on the maximum inlet concentrations of the individual components.

\section{References}

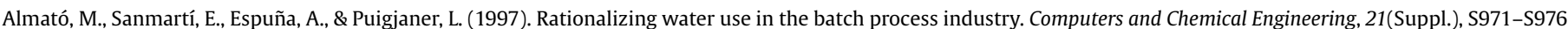

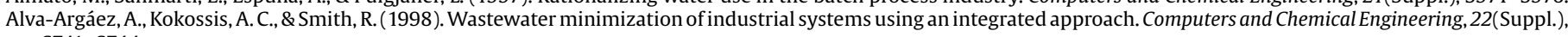
S741-S744.

Doyle, S. J., \& Smith, R. (1997). Targeting water reuse with multiple contaminants. Transactions of the IChemE, 75b, 181-189. 
Foo, D. C. Y., Manan, Z. A., \& Tan, Y. L. (2005). Synthesis of maximum water recovery network for batch process systems. Journal of Cleaner Production, 13 , $1381-1394$.

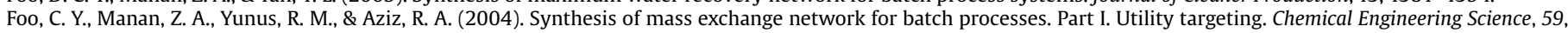
1009-1026.

Gouws, J. F., Majozi, T., \& Gadalla, M. (2008). Flexible mass transfer model for water minimization in batch plants. Chemical Engineering and Processing, 47, $2323-2335$.

Hallale, N. (2002). A new graphical targeting method for water minimization. Advances in Environmental Research, 6, 377-390.

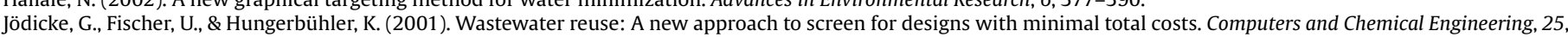
203-215.

Kim, J. K., \& Smith, R. (2004). Automated design of discontinuous water systems. Process Safety and Environmental Protection, 82, $238-248$.

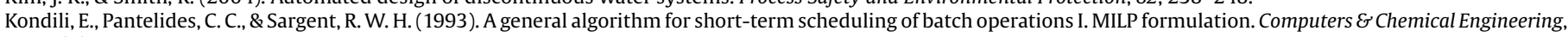
$17(2), 211-227$

Majozi, T. (2005). Wastewater minimization using central reusable storage in batch plants. Computers and Chemical Engineering, 29, $1631-1646$.

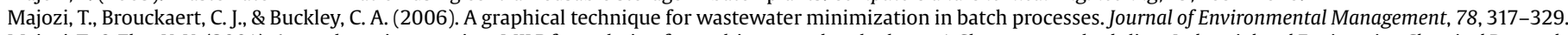

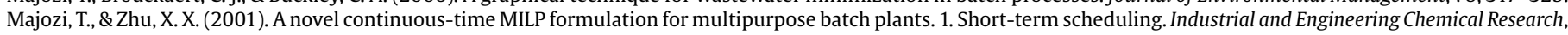
40,5935-5949.

Olesen, S. G., \& Polley, G. T. (1997). A simple methodology for the design of water networks handling single contaminants. Transactions of the IChemE, 75a, 420-426.

Quesada, I., \& Grossmann, E. I. (1995). Global optimization of bilinear process networks with multicomponent flows. Computers and Chemical Engineering, $19,1219-1242$.

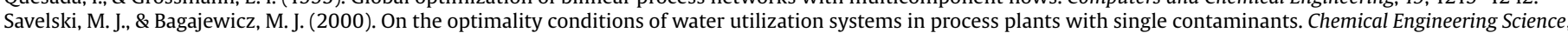
55, 5035-5048.

Wang, Y. P., \& Smith, R. (1994). Wastewater minimization. Chemical Engineering Science, 49, 981-1006.

Wang, Y. P., \& Smith, R. (1995a). Time Pinch Analysis. Transactions of the IChemE, 73a, 905-914.

Wang, Y. P., \& Smith, R. (1995b). Waste minimization with flowrate constraints. Transactions of the IChemE, 73, 889-904. 